\title{
Kilian Heck
}

\section{Mimesis versus Fiktion}

\section{Präfigurationen modernen Bildverständnisses bei Caspar David Friedrich}

Das zeichnerische Euvre Caspar David Friedrichs weist eine Fülle akribisch genau beobachteter Naturstudien auf. ${ }^{1}$ Es ist eine noch immer nicht abschließend geklärte Frage, weshalb Friedrich solchen Wert auf die Darstellung auch kleinster Details wie Baumnadeln oder Wurzelverästelungen legte. Werner Busch sieht diesen Detailismus in Zusammenhang mit der Vorliebe Friedrichs für geometrische Teilungsverfahren wie den Goldenen Schnitt, mit dem er eine ästhetische Strategie verfolgt habe, die den verlorenen Zusammenhang von Mensch, Natur und Gott erneut zum Vorschein bringen sollte. ${ }^{2}$ Solche akribische Befolgung bildnerischer Authentizität mag aber auch als Kritik anderer Verfahren verstanden werden, welche die fiktionale Bildgestaltung im Rahmen der Effektsteigerung beabsichtigten.

Für seine Gemälde griff Friedrich immer wieder auf diese Naturstudien zurück, die er aber in einen völlig neuen Zusammenhang stellte, indem er sie entsprechend seinen künstlerisch-ästhetischen Vorstellungen modifizierte. ${ }^{3}$ So kopierte Friedrich teilweise aus mehreren Naturstudien einzelne Bäume oder Äste und kompilierte sie in den Gemälden vollständig neu, so dass im fertigen Gemälde oft Einzelelemente zu finden sind, die aus dem in völlig anderem örtlichen und zeitlichen Zusammenhang entstandenen Studienmaterial entnommen sind.$^{4} \mathrm{Im}$ Folgenden soll untersucht werden, wie dieses zunächst merkwürdig anmutende Verfahren einer strikten Befolgung mimetischer Naturbeobachtung und fiktionaler Neukomposition dieser Teilfragmente im fertigen Werk einzuordnen ist. Hier sind vor allem zwei Diskurse innerhalb der Kunstdebatte des 18. Jahrhunderts zu erwähnen: zunächst der Illusionismus, der in der ersten Hälfte des 18. Jahrhunderts breit diskutiert wird. Gegen Ende des 18. Jahrhunderts tritt dann die Erörterung des Sublimen in den Vordergrund. Gerade die jüngste Forschung sieht Anzeichen dafür, dass Friedrich sowohl den Illusionismus wie auch das Erhabene

1 Vgl. dazu ausführlich Christina Grummt: Die Zeichnungen. Das gesamte Werk. München 2011, passim.

2 Vgl. Werner Busch: Caspar David Friedrich: Ästhetik und Religion. München 2003, S. $138 \mathrm{ff}$.

3 Vgl. Grummt: Die Zeichnungen (Anm. 1), S. 31.

$4 \mathrm{Zu}$ diesem Verfahren ausführlich Grummt: Die Zeichnungen (Anm. 1), S. 31; Johannes Grave: Caspar David Friedrich. München 2012, S. $173 \mathrm{ff}$. 
zu überwinden versuchte. ${ }^{5} \mathrm{Zu}$ beiden Konzepten bedarf es aber zunächst weiterer Ausführungen:

Mit seinen 1719 erschienenen Réflexions hat Jean-Baptiste Dubos die erste Theorie der ästhetischen Illusion vorgelegt, die eine Sinnestäuschung als Ursache des sinnlichen Vergnügens in der Kunst ausschließt. ${ }^{6}$ Dubos führt zur Unterlegung seiner Theorie das berühmt gewordene Beispiel an, dass jeder Betrachter ein Gemälde beurteilen könne, welches ein Ragout darstelle. So wie jeder in der Lage sei, kompetent den ,Wert' oder ,Unwert‘ eines Ragouts zu schmecken, so könne auch jeder Betrachter den Wert oder Unwert eines Gemäldes mit einem Ragout beurteilen. ${ }^{7}$ Der Betrachter eines solchen Bildes sei sehr wohl in der Lage, das mittels der Zentralperspektive konstruierte Bild als dargestellte Illusion zu erkennen und von der Wirklichkeit zu unterscheiden. ${ }^{8}$ Die Wahrnehmung des Kunstwerks erscheint bei Dubos demnach nicht mehr als eine Funktion der Vernunft, sondern als eine des Gefühls, und erreicht damit eine vollständige Relativierung in Bezug auf das betrachtende Subjekt. Die Illusion ist nicht Ursache des Vergnügens, sondern ihr Inzidenzpunkt. ${ }^{9}$

Diese Bindung der Illusion an das „sentiment“ und seine Distanzierung von der „raison“ ist eine Grundkonstante der Kunsttheorie des 18. Jahrhunderts. ${ }^{10}$ Das Paradoxon dieses Gedankens besteht nun darin, dass Kunst erst auf der Ebene der ästhetischen Illusion die Kompetenz besitzt, den einzelnen subjektiven Betrachter zu illudieren und epistemologisch mit dem Erfassen von Wirklichkeit $\mathrm{zu}$ betrauen. ${ }^{11}$ Der durch das Mittel der perspektivischen Konstruktion erreichte Illusionismus kann in Bildern des 18. Jahrhunderts demnach immer nur dann interpretiert werden, wenn solche Mittel als subjektiv-sinngebende Elemente nicht des Bildes, sondern des anschauenden Rezipienten verstanden werden.

Nun ist es kaum wahrscheinlich, dass Friedrich unmittelbar diese französische Illusionismusdiskussion rezipiert hat. Vielmehr wird in Bezug auf ihn immer wieder die Diskussion um das Erhabene angeführt, wobei insbesondere Schillers

5 Vgl. Grave: Caspar David Friedrich (Anm. 4), S. 194-199, bes. S. 198.

6 Vgl. Ludwig Tavernier: Apropos Illusion. Jean Baptiste Dubos' Einführung eines Begriffs in die französische Kunstkritik des 18. Jahrhunderts. In: Pantheon XLII (1984), S. 158-160, hier S. 158.

7 Vgl. ebd.

8 Vgl. ebd.

9 Vgl. ebd.

10 Vgl. Hubertus Kohle: Leidenschaft und kühler Blick. Diskursive kontra ästhetische Kunsttheorie im 18. Jahrhundert. In: Zeitschrift für Ästhetik und allgemeine Kunstwissenschaft XXXIII/2 (1988), S. 247-258, S. 251 f.

11 Vgl. Tavernier: Apropos Illusion (Anm. 6), S. 158. 
Aufsätze Vom Erhabenen (1793) und Über das Erhabene (1801) als kunsttheoretische Folgerungen aus Kants Begriffsbestimmungen zum Erhabenen genannt werden. ${ }^{12}$ Hierbei wird das gleiche Problem wie bei Dubos virulent, aber ins Gegenteil gewendet. Sobald nämlich der Betrachter vergegenwärtige, dass er einer künstlichen Inszenierung beiwohne, gebe es für ihn keine Veranlassung mehr, das Geschehen auf der Bühne tatsächlich als bedrohlich wahrzunehmen. ${ }^{13}$ Die Rezeption des um 1800 in den kunsttheoretischen Schriften vielfältig diskutierten Problems des Erhabenen hat ebenfalls diesen Mangel konstatiert, wobei insbesondere Christian August Semler und Carl Ludwig Fernow zu nennen sind. Beide haben in ihren Traktaten Über die höchste Vollkommenheit in den Werken der Landschaftsmalerei (1800) und Über die Landschaftsmalerei (1803) die Kategorie des Erhabenen im Nachklang Schillers als wenig probates Mittel für die Landschaftsmalerei gewertet. ${ }^{14}$ Die Frage nach der Erkennbarkeit fiktionaler Bildinhalte steht demnach sowohl bei der französischen Illusionismusdebatte wie auch bei der deutschen Diskussion um das Erhabene um 1800 im Zentrum der Erörterung.

Im Zusammenhang mit Friedrich hat insbesondere Johannes Grave mehrfach die Kritik des Künstlers an der Ästhetik des Erhabenen betont. ${ }^{15}$ Grave führt als Grund die lutherische Religiosität Friedrichs an, deretwegen er in der Erfahrung des Erhabenen einen Akt der Hybris habe sehen müssen: ${ }^{16}$

Alle Teuschung macht einen widrigen Eindruck, wie aller Betrug. Z. B. Wachsfiguren werden immer etwas zurückstoßendes haben je teuschender sie gemacht sind. Ein Bild muss sich als Bild als Menschenwerk gleich darstellen; nicht aber als Natur täuschen wollen. Doch strebet immer hin, ihr Mahler nach Wahrheit, wahrhaftiglich teuschen werdet ihr doch nie und ist auch nicht Forderung der Kunst [...]. ${ }^{17}$

Ausgehend von diesem späten, etwa 1828 bis 1830 entstandenen und in der Friedrich-Forschung seit langem bekannten Text Äußerungen bei Betrachtung einer Sammlung von Gemählden von größtentheils noch lebenden und unlängst ver-

12 Vgl. Grave: Caspar David Friedrich (Anm. 4), S. $194 \mathrm{f}$.

13 Ebd.

14 Ebd., S. 195. - Bei Fernow heißt es etwa: „Die bildende Kunst kann durch ihre Darstellungen das Gefühl des Erhabenen nicht unmittelbar wecken, wie die Natur; denn sie kann das Erhabene nicht wirklich darstellen, wie diese“, zit. nach Grave, ebd., S. 195.

15 Vgl. ebd., S. 194-199, bes. S. 198.

16 Vgl. ebd., S. 198.

17 Caspar David Friedrich: Äußerungen bei Betrachtung einer Sammlung von Gemählden von größtentheils noch lebenden und unlängst verstorbenen Künstlern. Bearb. v. Gerhard Eimer mit Günter Rath. Frankfurt a.M. 1999, S. 86; teilweise auch zit. bei Grave: Caspar David Friedrich (Anm. 4), S. 198. - Für den Hinweis auf diese Bemerkung Friedrichs bin ich Johannes Grave zu Dank verpflichtet. 
storbenen Künstlern kann ein gewisses Misstrauen Friedrichs gegen optische Illusion angenommen werden. ${ }^{18}$

Nun gibt es einige wenige Äußerungen Friedrichs, die in seinen Werkprozess Einblick gewähren: So ist seine Ablehnung von Vorzeichnungen wie etwa Farbstudien bekannt, wie sie von der Plein-air-Malerei um Pierre-Henri de Valenciennes propagiert wurden. Friedrich äußert sich hierzu wie folgt:

Wie sehr man auch die Weise in Schutz nimmt das beabsichtigte ins Große auszuführende Bild zuvor ins Kleine zu mahlen, so bin ich doch dagegen; denn man kopirt nur zu leicht sich selbst und die freie geistige Nachbildung der Natur, das eigendliche Schaffen, hört auf. ${ }^{19}$

Entsprechend finden sich farbige Vorzeichnungen in seinem Werk eher selten. ${ }^{20}$ Der für Friedrich charakteristische Typus der „bildmäßigen Zeichnung“ ist hier nicht im strengen Sinne als Vorzeichnung zu verstehen, da sie weitgehend ausgearbeitet ist. ${ }^{21}$

Der nicht auf klassischen Vorzeichnungen beruhende Werkprozess des Künstlers ist oft beschrieben worden: Friedrich entwickelt eine Art Kompositverfahren, das zwar den einzelnen Gegenstand, etwa einen Baum, minutiös in der Zeichnung wiedergibt, der aber etwa beim Übertrag des gezeichneten Baumes auf ein Gemälde wie bei einem Pasticcio die Dinge aus den verschiedensten Kontexten zusammenaddiert. ${ }^{22}$ Daraus ergibt sich wiederum die Konsequenz, dass je Bild eine neue Ansicht eröffnet wird, die nicht durch das bildnerische Objekt selbst die Rezeption reguliert, sondern es dem Betrachter überlässt, die disparaten Einzeldinge zusammenzufügen. Freilich wäre hier einzuwenden, dass Friedrich selbst es ist, der diese Einzeldinge im Rahmen einer großen, neuen Gesamtkomposition zusammenfügt. Dennoch ist es kaum zu erklären, warum er einerseits so sehr dem mimetischen Detailreichtum eines jeden Blattes huldigt, andererseits aber in geradezu kühner Weise eben nicht die ,Wirklichkeit‘ der Dinge, ihre äußere Realität,

18 Einschränkend wäre hier zu bemerken, dass Friedrich auch Transparentbilder schuf, die nur mittels künstlerischer Beleuchtung als optische Illusion überhaupt funktionieren konnten, vgl. hierzu Birgit Verwiebe: Lichtspiele. Vom Mondscheintransparent zum Diorama. Stuttgart 1997, S. 59-62.

19 Friedrich: Äußerungen (Anm. 17), S. 27: „Wie sehr man auch die Weise in Schutz nimmt das beabsichtigte ins Große auszuführende Bild zuvor ins Kleine zu mahlen, so bin ich doch dagegen; denn man kopirt nur zu leicht sich selbst und die freie geistige Nachbildung der Natur, das eigendliche Schaffen, hört auf.“

20 Vgl. auch die wenigen Beispiele für farbige Vorzeichnungen, die nicht als „bildmäßig“ gelten, bei Grummt: Die Zeichnungen (Anm. 1), etwa S. 580, Nr. 606.

21 Vgl. ebd., S. 30.

22 Vgl. Busch: Caspar David Friedrich (Anm. 2), S. 82-85; Grummt: Die Zeichnungen (Anm. 1), S. $31 \mathrm{f}$. 
sondern vielmehr ein Komposit einzelner innerer und äußerer Bilder wiedergibt. Johannes Grave hat denn auch die Herauslösung der datierten und geographisch markierten Zeichnungen aus ihrem Zusammenhang für Friedrich nicht in erster Linie als Ausdruck mimetischer Genauigkeit gewertet, sondern als „Gegengewicht zu seinen bisweilen irreal anmutenden, strengen Bildkompositionen“. ${ }^{23}$ Erst hierdurch sei ein spannungsreiches Wechselspiel zwischen Wirklichkeit und tieferer Bedeutung erreicht, so Grave. ${ }^{24}$

Einige Beispiele können das angesprochene Verfahren erläutern: Werden etwa die Vorstudien der Abtei im Eichwald (1809/10) mit dem ausgeführten Gemälde verglichen, dann ergeben sich einige signifikante Veränderungen (Abb. 1 und 2). ${ }^{25}$ Aus der Studie einer Eiche vom 5. Mai $1809^{26}$ verwendet Friedrich ebenso Teilfragmente wie aus der nur Wochen später angefertigten Studie von Weinlaub und Buchen vom 13./14. Juni 1809 (Abb. 3 und 4). ${ }^{27}$ Zunächst findet sich der Baum der Zeichnung auf dem Gemälde fast exakt wieder, direkt links neben der Ruine. Allerdings ist auf dem Gemälde ein weiterer starker Ast links des Baumes zu erkennen. Dieser Ast findet sich nicht auf der Zeichnung vom 5. Mai, wohl aber auf der vom 13. Juni wieder. Folgendes war geschehen: Der kleine, in der Mitte befindliche Baum in der unteren Reihe wurde von Friedrich interessanterweise in Gänze in seiner zickzackförmigen Gesamtgestalt kopiert, um dann als linker Ast wieder im Gemälde aufzutauchen. ${ }^{28}$ Dass Friedrich hier aus zwei in ihrer Gestalt vollständig entwickelten Bäumen der beiden Zeichnungen in einer Art additivem Verfahren einen völlig neuen Baum auf dem Gemälde kreiert, ist bereits für sich bemerkenswert. Dass er aber einmal im Gemälde die unvollständige Gesamtgestalt der einen zeichnerischen Vorlage - der vom 5. Mai - übernimmt und dann für den noch fehlenden linken Ast wiederum die Gesamtgestalt eines anderen Baumes aus der Zeichnung vom 13. Juni überträgt, diesen Baum aber zum Ast modifiziert, ist ein Indiz dafür, dass Friedrich hochkomplexe „Kompositbäume“ schafft, die eben nicht mehr der mimetischen Übernahme aus der Vorlage verpflichtet sind. ${ }^{29}$

23 Grave: Caspar David Friedrich (Anm. 4), S. 177.

24 Vgl. ebd., S. 178.

25 Vgl. Helmut Börsch-Supan u. Karl Wilhelm Jähnig: Caspar David Friedrich: Gemälde, Druckgraphik und bildmäßige Zeichnungen. München 1973, S. 304, Nr. 169.

26 Vgl. Grummt: Die Zeichnungen (Anm.1), S. 558, Nr. 586. - Bei Grummt finden sich auch frühere Nachweise dieser Übernahme aus der genannten Zeichnung in der Sekundärliteratur; vgl. auch Busch: Caspar David Friedrich (Anm. 2), S. 77 f., und den Kommentar von Grummt (Anm. 1) zu Buschs Beobachtung, S. 558, Nr. 586.

27 Vgl. ebd., S. 570 , Nr. 594.

28 Vgl. ebd., S. 31 f.; S. 558.

29 Dieser Begriff bei Grummt, ebd., S. 31. 


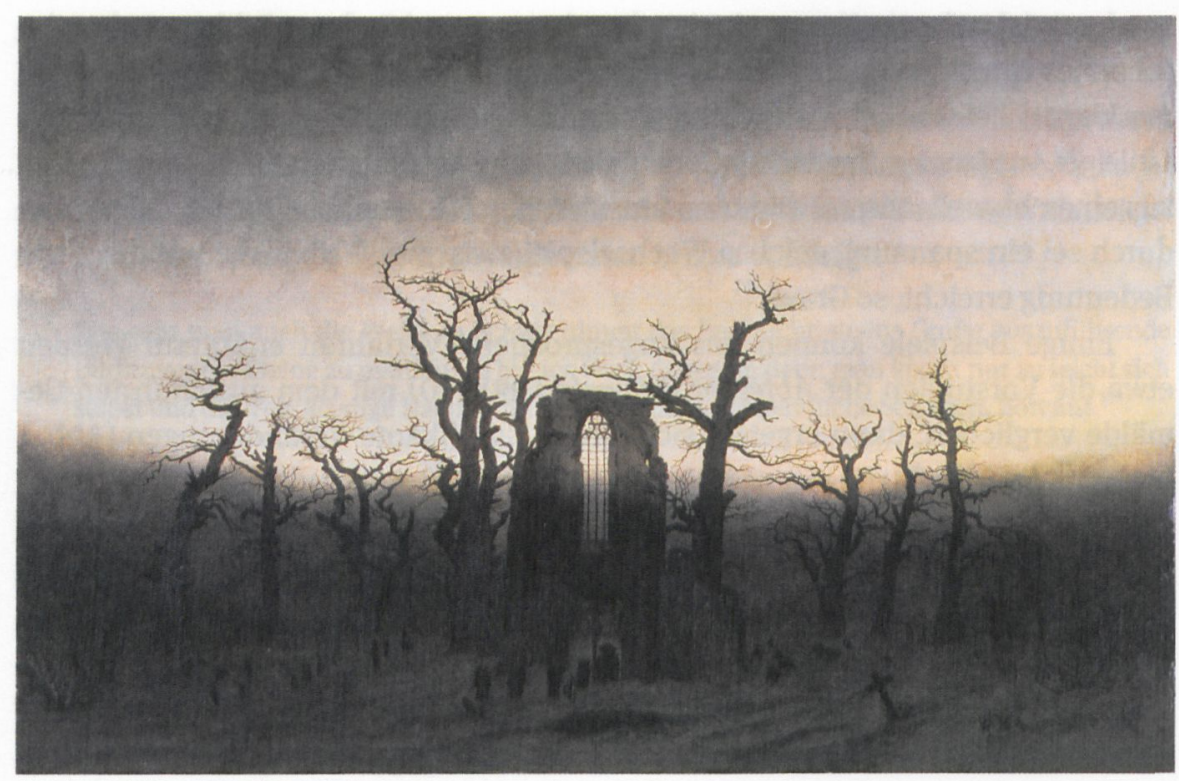

Abb. 1: Caspar David Friedrich, Abtei im Eichwald, 1809-10, Öl auf Leinwand. Berlin, Staatliche Museen zu Berlin, Alte Nationalgalerie.

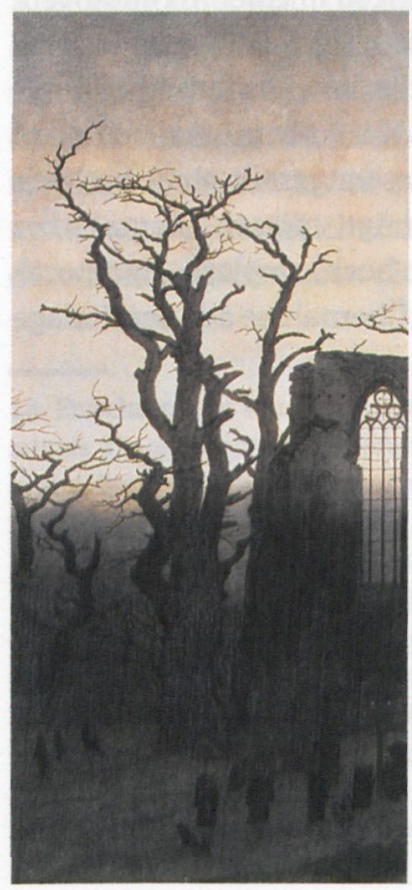

Abb. 2: Caspar David Friedrich, Abtei im Eichwald (Detail). 


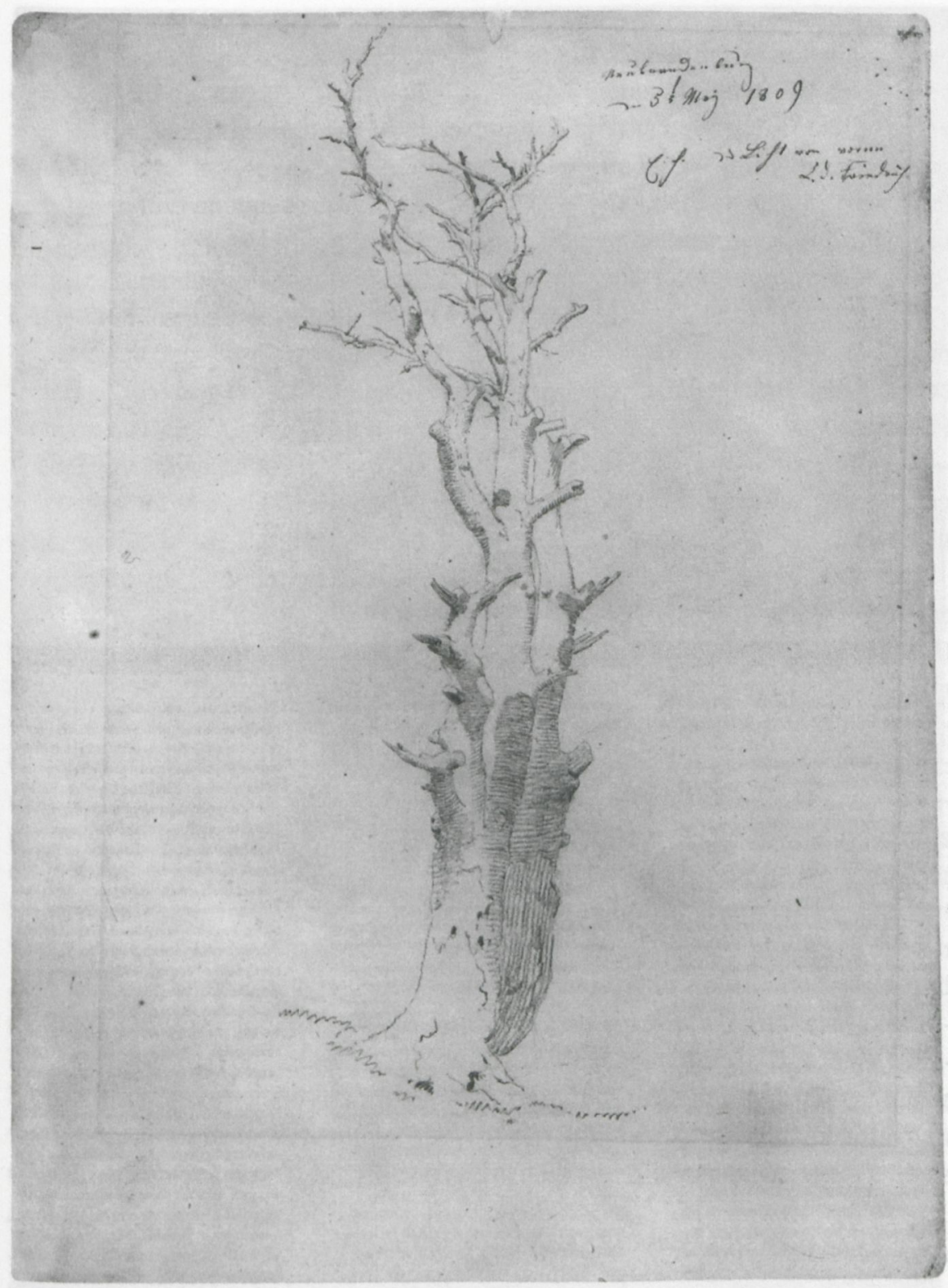

Abb. 3: Caspar David Friedrich, Studie einer Eiche, 5. Mai 1809, Bleistift. Nürnberg, Germanisches Nationalmuseum. 


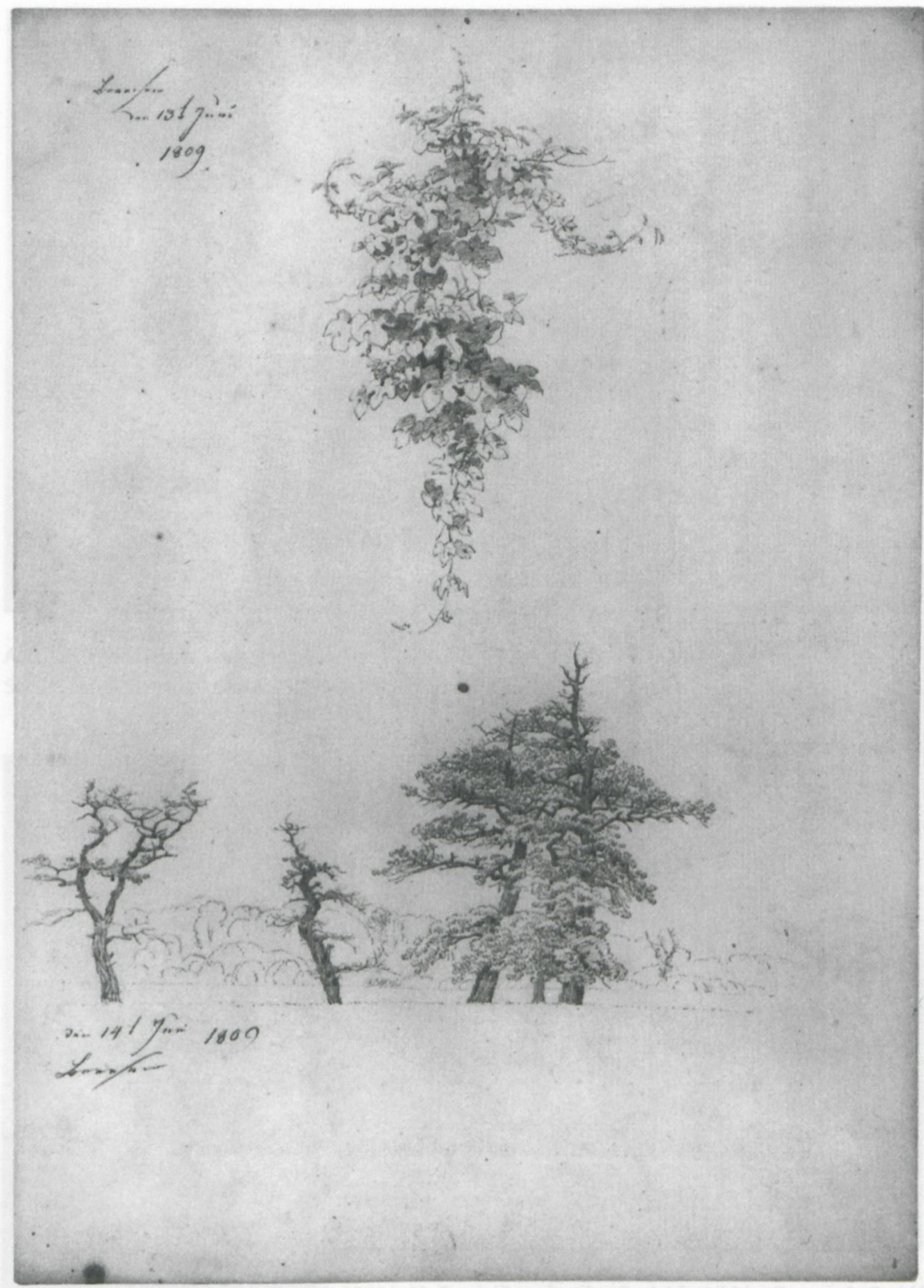

Abb. 4: Caspar David Friedrich, Studie von Weinlaub und Buchen vom 13./14. Juni 1809, Bleistift. Oslo, Nationalmuseum. 
Der Grund für dieses Verfahren ist relativ klar: Friedrich musste es bei der aus dem Halbdunkel aufscheinenden Abtei primär auf die Silhouette ankommen, bei der das Licht die Architektur und die Bäume von hinten umfängt. Ein nach vorne zum Betrachter ragender Ast wie auf der Zeichnung hätte im Gemälde seine Wirkung verfehlt. Genau dieses Verfahrens, einzelne Bildelemente auseinanderzunehmen und montageartig ganz neu zu formatieren, bedient sich Friedrich auf vielfältigste Weise, wie an dutzenden Beispielen $\mathrm{zu}$ belegen wäre. ${ }^{30}$ So werden auch in anderen Gemälden gegenüber den Vorgaben der Zeichnungen völlig neuartige Kompositbäume geschaffen.

Mit solchen subtilen Beobachtungen haben Grummt und auch Grave jeder Untersuchung zu Friedrich wichtige Impulse gegeben. Denn hier kehrt nichts weniger als eine Grundfrage der Forschung zu dem Greifswalder Romantiker wieder: diejenige nämlich, ob der Künstler trotz aller Ummontierungen der Einzelszenen am Mimetischen insgesamt festhält - oder ob er mitunter den Weg zu einer völlig freien Formenfindung wählt, die das Naturvorbild wenigstens in einigen Details fiktional ergänzt.

Ein weiteres Beispiel für ein Kompositverfahren bei Friedrich wäre das Gemälde Die Schwestern auf dem Söller am Hafen/Nacht im Hafen von 1820 (Abb. 5). ${ }^{31}$ Hier finden sich gleich mehrere Architekturen vereint: der Rote Turm und die Marktkirche aus Halle, das Stralsunder Rathaus, ein Turm aus Neubrandenburg sowie die Silhouette des Greifswalder Hafens. ${ }^{32}$ Dieses aus den verschiedensten, keineswegs nur von Friedrich selbst stammenden Bildvorlagen komponierte Gemälde macht sein Kompositverfahren nicht nur offensichtlich, es erklärt es geradezu zum Programm. Die Künstlichkeit des Bildwerkes wird hier insofern aufgegriffen, als jedem Betrachter die fiktionale Struktur sofort evident werden muss. Die Stadt wird zum Traum, zur Erscheinung, zur Vision, die ein „Vollkommenes, bildmäßiges Arrangement der Architekturansichten“ zeigt und gerade nicht anstrebt, dass der Betrachter die ausschließlich bildliche Vermittlung vergisst. ${ }^{33}$ Insbesondere für dieses Gemälde macht Werner Busch im Hamburger Katalog von 2006 jedoch geltend, dass hier trotz der Zusammenfügung von Architekturen aus den unterschiedlichsten örtlichen Kontexten unbedingt an der mimetischen Ausrichtung jedes einzelnen Elements festgehalten wird. ${ }^{34}$ Diese

30 Weitere Beispiele bei Grave: Caspar David Friedrich (Anm. 4), S. 178ff., und Grummt: Die Zeichnungen (Anm. 1), S. $31 \mathrm{f}$.

31 Börsch-Supan u. Jähnig: Caspar David Friedrich (Anm. 25), S. 358, Nr. 263.

32 Vgl. Grave: Caspar David Friedrich (Anm. 4), S. 216.

33 Ebd.

34 Vgl. Werner Busch: Friedrichs Bildverständnis. In: Caspar David Friedrich: die Erfindung der Romantik (Museum Folkwang, Essen, 5. Mai bis 20. August 2006, Hamburger Kunsthalle, 7. Oktober 2006 bis 28. Januar 2007), München 2006, S. 32-47, S. 39 f. 


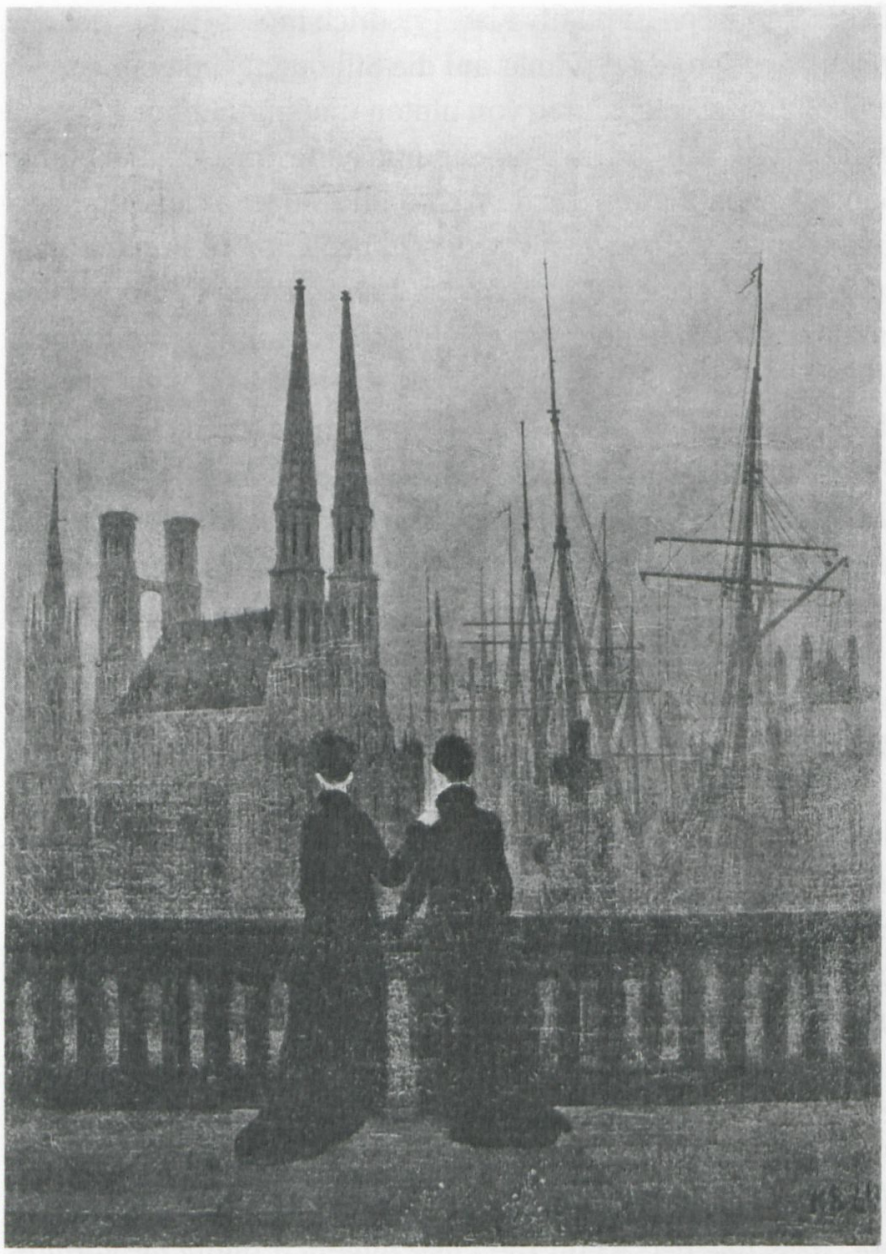

Abb. 5: Die Schwestern aufdem Söller am Hafen/Nacht im Hafen, 1820. St. Petersburg, Eremitage.

Grundüberlegung scheint zwar auf dieses Werk beziehbar, ist jedoch nicht mehr ohne Einschränkung auf das Gesamtwerk Friedrichs anzuwenden:

Um bei der Beantwortung der Frage der mimetischen Nähe oder Ferne ein Stück weiterzukommen, sei auf das Osloer Blatt mit drei Kiefernstudien verwiesen. Bei diesem Blatt wird die Bedeutung der Beobachtung im Werkprozess Friedrichs direkt physisch erfahrbar: So zeichnete Friedrich besagte drei Kiefern an ein und demselben Tag, dem 13. April 1807 (Abb. 6). ${ }^{35}$ Oben links beginnend,

35 Vgl. Grummt: Die Zeichnungen (Anm. 1), S. 496f., Nr. 531. 


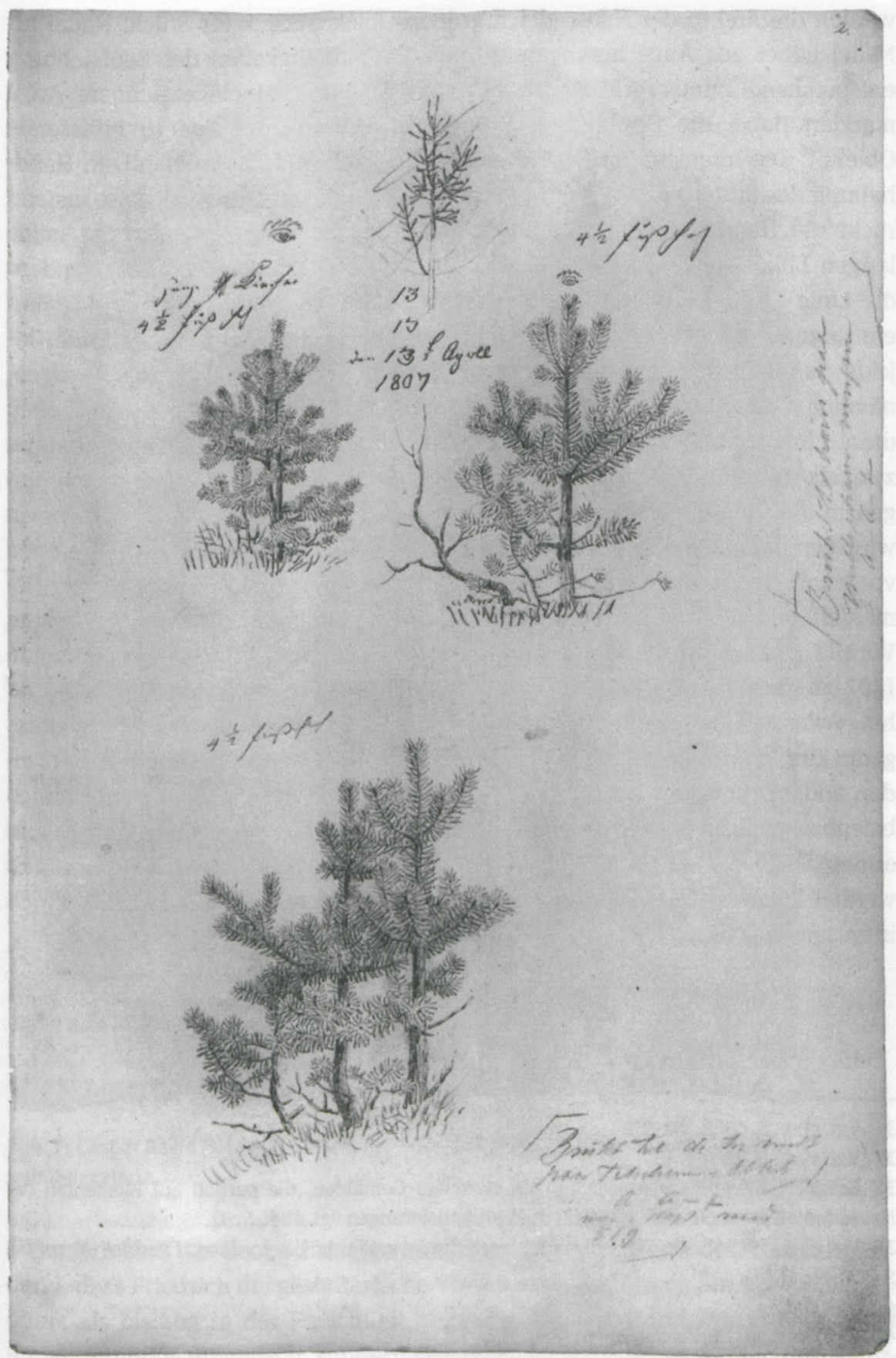

Abb. 6: Caspar David Friedrich, Kiefernstudien, 13. April 1807, Bleistift. Oslo, Nationalmuseum. 
werden die drei in der Natur gleich großen Objekte Stück für Stück, Nadel für Nadel näher ans Auge herangeholt und die Feinteiligkeiten der Beobachtung entsprechend immer größer. Das stilisiert ins Blatt hineingezeichnete Auge markiert dabei die Position des Künstlers in Bezug auf das zu erfassende Objekt. ${ }^{36}$ Die Intensität der Naturbeobachtung geht mit der vorsichtigen Handhabung des Stiftes einher. Nicht in einem Schwung, sondern stückweise tastend rückt die Hand mit dem Bleistift auf dem Papier voran. ${ }^{37}$ Es werden keine langen Linien gezogen, die den Gegenstand grob skizzieren würden, sondern die Linie erzeugt ihrerseits die Umrissform des Gegenstandes. Das ist insofern ein ungewöhnliches, wenn auch nicht gänzlich neues Verfahren, weil die Objekte damit nicht nur etwas erhalten, was sie in Wirklichkeit nicht besitzen, nämlich Kontur, sondern weil sie zugleich auch substantiell erst durch eben diese Linie erzeugt werden. Außerdem werden, und das hat Christina Grummt zweifelsfrei nachgewiesen, nicht nur Kompositbäume von einzelnen Zeichnungen in die Gemälde übertragen. Es werden darüber hinaus, wenn auch nur in winzigen Details, Modifikationen zwischen den Naturstudien und den Gemälden vorgenommen, also aus den Naturstudien übernommene Elemente in den Gemälden gelängt. ${ }^{38}$ So hat die erwähnte Kiefernstudie von 1807 eben auch zum Vorbild gedient für die kleinen Kiefern, die in Friedrichs Tetschener Altar von $1807 \mathrm{zu}$ sehen sind (Abb. 7 und 8). ${ }^{39}$ Jedoch hat schon Börsch-Supan darauf hingewiesen, dass die Kiefer in der Zeichnung gegenüber dem Gemälde etwas geduckter, niedriger erscheint. ${ }^{40}$ Friedrich hat also - und das wäre mit Dutzenden anderer Beispiele durch das neue Werkverzeichnis von Grummt zusätzlich belegbar - tatsächlich mitunter die Bäume gelängt, hat Nadeln hinzugefügt. ${ }^{41}$ In anderen Fällen, wie beim Einsamen Baum, geschieht es gerade umgekehrt. Es werden Elemente wie das Storchennest weggenommen und der Baum dadurch insgesamt gekürzt. ${ }^{42}$

36 Vgl. ebd., S. $496 f .$, Nr. 531.

37 Vgl. ebd., S. 32.

38 Beispiele für größere Abweichungen zwischen Gemälden, die partiell auf Elementen der Naturstudien beruhen, ebd., S. 31; für die Vertikalisierungen vgl. ebd., S. 32.

39 Vgl. ebd., S. 496f., Nr. 531; vgl. auch Börsch-Supan u. Jähnig: Caspar David Friedrich (Anm. 25), S. 300, Nr. 167.

40 Vgl. ebd.

41 Vgl. Grummt: Die Zeichnungen (Anm. 1), S. 32.

42 Vgl. Börsch-Supan u. Jähnig: Caspar David Friedrich (Anm. 25), S. 378, Nr. 298; Grummt: Die Zeichnungen (Anm. 1), S. 451, Nr. 481. 


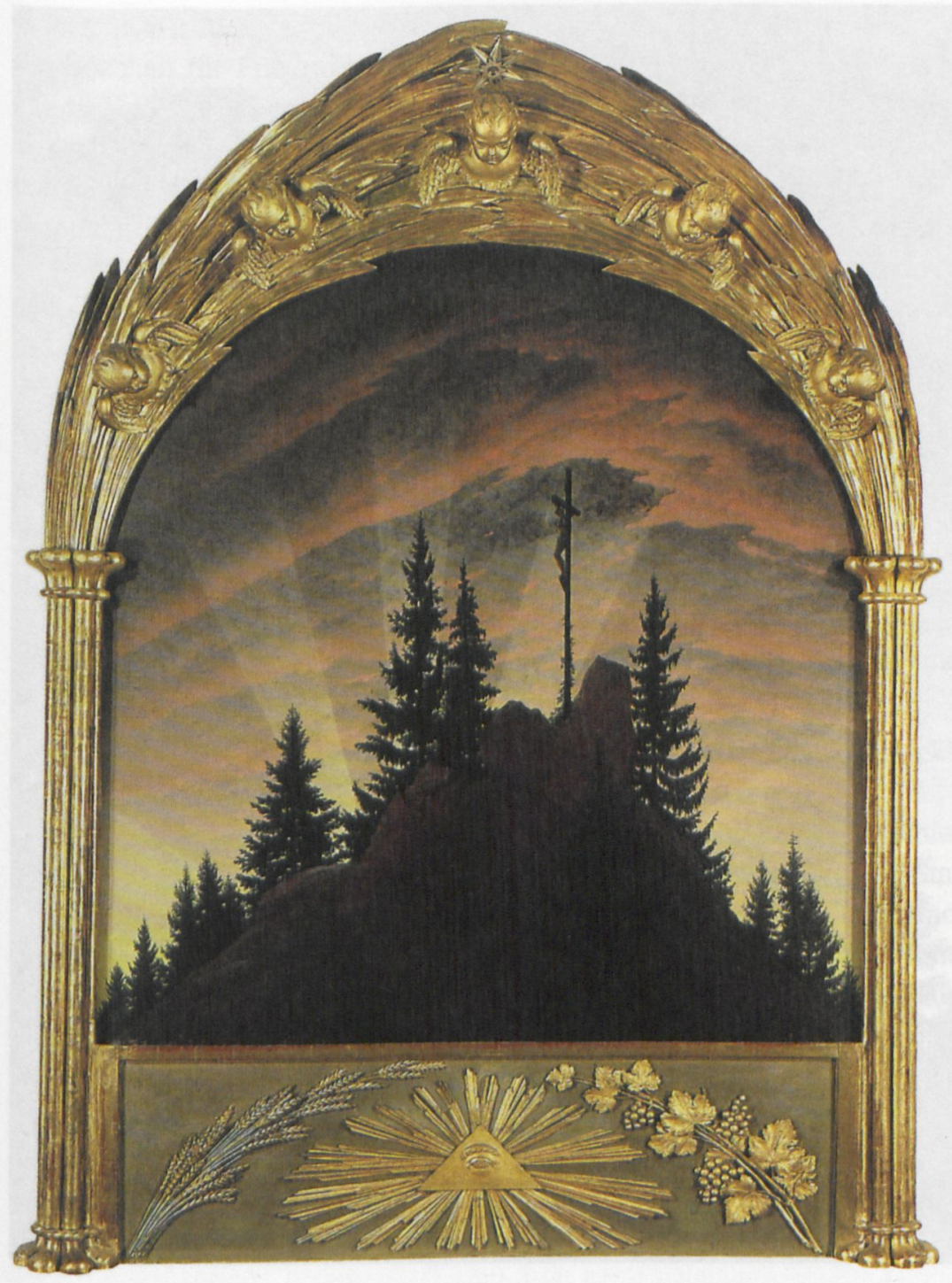

Abb. 7: Caspar David Friedrich, Tetschener Altar, 1807-08, Öl auf Leinwand. Dresden, Gemäldegalerie.

Sicherlich sind das Beobachtungen an kleinen Details. Aber es ist eben entscheidend, dass Friedrich die gestalterische Vorgabe der Zeichnung für das Gemälde öfters als bislang in der Forschung angenommen - abwandelt und eben nicht immer minutiös mimetisch, sondern gelegentlich durchaus großzügig das Naturvorbild von der Zeichnung auf das Gemälde überträgt. Die Aussage, es handele 


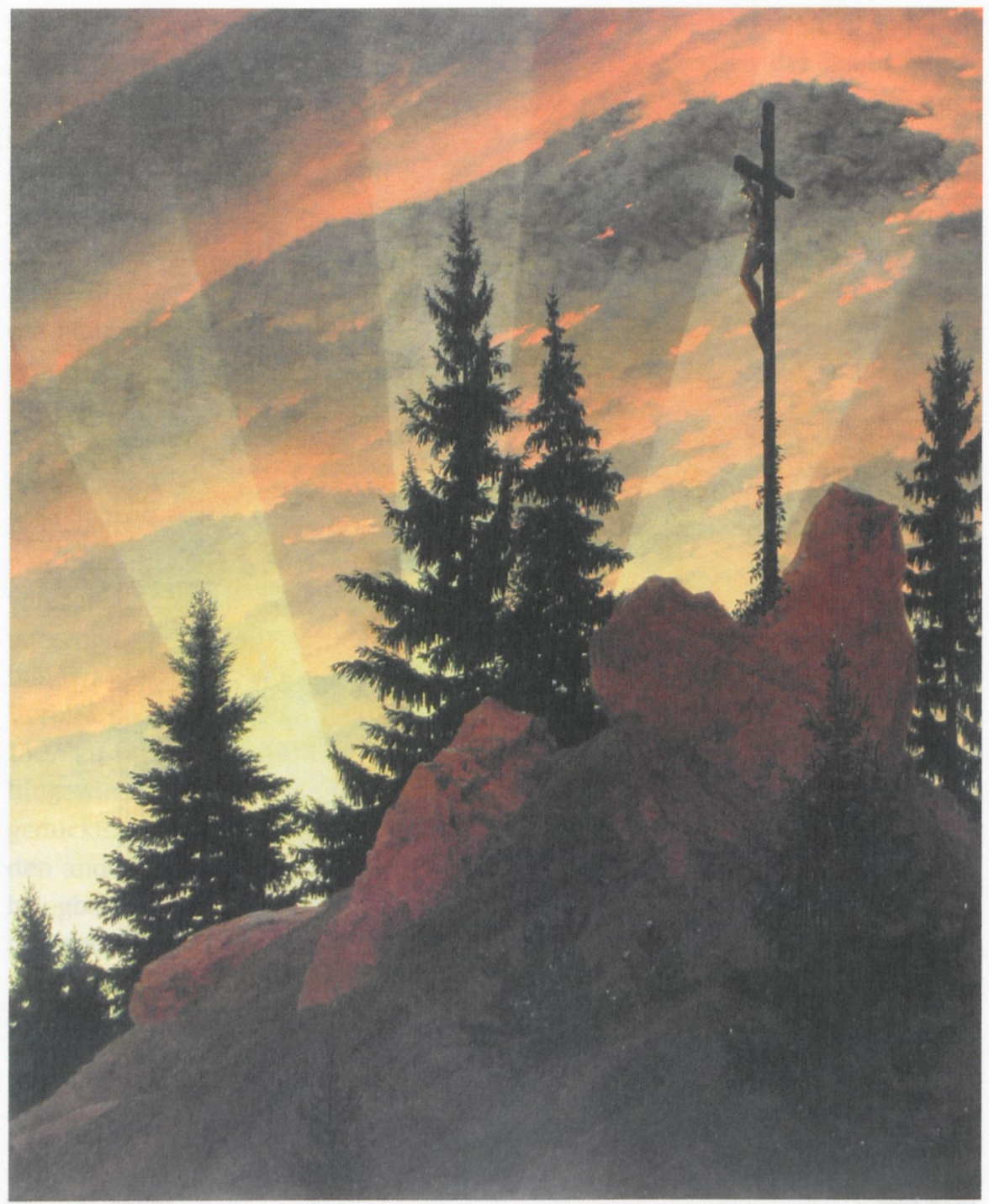

Abb. 8: Caspar David Friedrich, Tetschener Altar (Detail).

„sich bei Friedrich geradezu um einen Authentizitätszwang, der die Wahrheit des auf der Welt Erscheinenden offenbar aus religiöser Überzeugung verbürgen muss“, ist daher mit Einschränkungen zu versehen. ${ }^{43}$

43 Busch: Friedrichs Bildverständnis (Anm. 34), S. 40. 
Es wäre jedoch verfehlt, wenn diese fiktionale Ergänzungsstrategie zu einem Kennzeichen für Friedrichs Prämodernität gemacht würde. Denn die Zahl der Landschaftsmaler, die einen wesentlich freieren Umgang mit den Vorgaben der Natur in ihren Gemälden pflegen, ist Legion. Hier würden etwa die Landschaften von Jakob Philipp Hackert einen reichen Fundus abgeben. Es scheint vielmehr die Kombination von zweierlei zu sein, das bei Friedrich als Besonderheit auffällt: zum einen besagte Treue zu einer Natur, die aber immerhin in Teilbereichen fiktional ergänzt werden kann, zum anderen eine besondere Perspektivität, die vor allem Johannes Grave und Hilmar Frank beschäftigt hat: ${ }^{44}$ Johannes Grave hat in seiner Studie von 2001 der einfachen Übertragung philosophischer Denkkategorien auf Friedrichs Eismeer, ${ }^{45}$ eines seiner berühmtesten Bilder von 1823, widersprochen:

Doch die Bildstruktur und Ikonographie des Eismeers lassen den ausgelösten ,Mechanismus' nicht an sein eigentliches Ziel gelangen. Auf den zweiten Blick offenbart sich nicht nur die Hilflosigkeit des Zuschauers, dessen Standort in dem vom Bild beschriebenen Raum imaginiert wird, sondern auch das Problem, dass sich der konkrete Betrachter wegen der perspektivischen Uneindeutigkeiten nicht in gewohnter Weise zum Bild hin positionieren kann. Der Betrachter sieht nun sich in Frage gestellt; das Dargestellte, der Schiffbruch, wird zweitrangig. ${ }^{46}$

Es liegt auf der Hand, dass diese Bezugnahme auf das betrachtende Subjekt einen auf Mehrdeutigkeit angelegten Bildgehalt zugrunde legen muss.

Hilmar Frank schlägt eine ähnliche Richtung in seinem 2004 erschienenen Buch zur Perspektivität bei Friedrich vor. Auch er unterstreicht die Standortgebundenheit von Friedrichs Kunst und betont, dass es bei der Werkbetrachtung nach seiner Auffassung auch jeweils andere Punkte geben müsse, von denen aus das Dargestellte beobachtet werden kann. ${ }^{47}$ Frank beschreibt den Fluchtpunkt als eine visuelle Metapher, ${ }^{48}$ die er wie folgt erläutert:

Es ist eine Grundeinsicht des perspektivischen Denkens, daß jede Perspektive nur eine Teilwahrheit erlangt, daß sie gleichermaßen berechtigt wie begrenzt ist, daß daher die Perspektiven einander ergänzen und folglich dort, wo es zum Konflikt der Perspektiven kommt, nicht davon die Rede sein kann, alle Relevanz sei auf EINER Seite zu finden. ${ }^{49}$

44 Vgl. Johannes Grave: Caspar David Friedrich und die Theorie des Erhabenen. Friedrichs „Eismeer" als Antwort auf einen zentralen Begriff der zeitgenössischen Ästhetik. Weimar 2001; Hilmar Frank: Aussichten ins Unermeßliche. Perspektivität und Sinnoffenheit bei Caspar David Friedrich. Berlin 2004.

45 Vgl. Börsch-Supan u. Jähnig: Caspar David Friedrich (Anm. 25), S. 386f., Nr. 311.

46 Vgl. Grave: Caspar David Friedrich und die Theorie des Erhabenen (Anm. 44), S. 127.

47 Vgl. Frank: Aussichten ins Unermeßliche (Anm. 44), S. 4.

48 Vgl. ebd., S. 6.

49 Ebd., S. 88. 
Frank führt weiter zu Friedrich aus:

Mit der Tiefenperspektive ins Offene findet er zu jener Raumauffassung, welche die Setzungen des Glaubens nicht mehr buchstäblich vollzieht, sondern dem Betrachter des Bildes suggeriert - oder bloß anheimstellt [...]..$^{50}$

Dieses Herausnehmen der Bilddeutung aus dem Gemälde und ihre Überantwortung an den Künstler oder Betrachter ist demnach eindeutiger Befund. Oder noch anders ausgedrückt: Die möglichst eindeutige (symbolische) Ausdeutung der wie Embleme herausgelesenen Inhalte im Bild dominiert die Interpretation so stark, dass nicht mehr darauf geachtet wird, dass aufgrund eines absichtlich durch Friedrich verunklärten perspektivischen Betrachterstandortes eine eindeutige Sinnfestschreibung erst außerhalb des Bildes durch den Betrachter erfolgen kann. Dass dieses Relativitätsdenken durch den subjektiven perspektivischen Standort des Betrachters vor dem Bild unmittelbar bestimmt ist, würde sich in die - von Friedrich abgelehnten - Jenenser frühromantischen Kategorien einfügen, in den „ganzen Kosmos der frühromantischen Fragmenterfahrung des Ich vom Verlust der Ganzheit und der Sehnsucht nach universalem Zusammenhang “. ${ }^{51}$ Indes scheint das perspektivische, auf einen subjektiven Betrachter bezogene Sehen weit früher in das 18. Jahrhundert zurückzugehen, wie zu Anfang dieses Beitrags ausgeführt. ${ }^{52}$ Es lässt sich demnach eine lange und intensive Tradition der Kritik am illusionsgebundenen Bild feststellen, die bei Friedrich zumindest im Zusammenhang mit seiner Kritik am Erhabenen nachweisbar ist und gut zu seinem zutiefst lutherischen Denken samt dessen Ablehnung äußerer Bilder, die immer auch täuschende Bilder sind, zugunsten des inneren Bildes passen würde. ${ }^{53}$

Die entscheidende Rolle des betrachtenden Subjekts wird auch in einer anderen Quelle formuliert, die Hilmar Frank erstmals auf Caspar David Friedrich bezogen hat. ${ }^{54}$ In der Allgemeinen Geschichtswissenschaft von Johann Martin Chladenius von 1752 beschreibt der Autor das gleichberechtigte Nebeneinander verschiedener Perspektiven auf ein und dieselbe Sache:

Es gibt einen Grund, warum wir die Sache so, und nicht anders erkennen: und dieses ist der Sehe-Punkt von derselben Sache [...]. Aus dem Begriff des Sehe-Punkts folget, daß Personen,

50 Ebd., S. 92.

51 Busch: Caspar David Friedrich (Anm. 2), S. 13.

52 Zudem hat Friedrich bekanntlich den Jenaer Idealismus eher abgelehnt, so dass ein anderer Zusammenhang gesucht werden sollte.

53 Hierzu Johannes Grave: Caspar David Friedrich. Glaubensbild und Bildkritik. Zürich 2011, besonders S. $33 \mathrm{ff}$.

54 Vgl. Frank: Aussichten ins Unermeßliche (Anm. 44), S. 6. 
die eine Sache aus verschiedenen Sehe-Punkten ansehen, auch verschiedene Vorstellungen von der Sache haben müssen [...]. ${ }^{55}$

Chladenius, den Reinhart Koselleck zu einem Kronzeugen des auf das Subjekt bezogenen Geschichtsmodells um 1800 macht, ${ }^{56}$ also für den historischen Relativismus und damit für die Zeitabhängigkeit jedweder Geschichtsbetrachtung in Anspruch nimmt, könnte als Erklärungsmodell auch bei Friedrich greifen - ohne dass wir freilich wissen, ob Friedrich Chladenius gelesen hat.

Zusammenfassend für die auf den letzten Seiten angestellten Überlegungen ließe sich festhalten, dass Friedrich die mimetische Darstellung der Dinge, insbesondere der Naturdinge als Gottes Schöpfung, zunächst deswegen so stringent verfolgte, weil er damit nicht zuletzt auch eine Kritik der perspektivischen Illusion vornehmen wollte. Daher erklärt sich die minutiöse Darstellung selbst der kleinsten Fichtennadeln und Kiefernzweige. Gleichwohl hat er nicht gezögert, Einzelszenen aus den verschiedensten Kontexten und Lokalitäten im Bild zu synthetisieren. Soweit zu dem in der Forschung längst bekannten Verfahren. Dass aber beides, strengste Naturtreue einerseits und fiktionales Kompositverfahren andererseits, in den Gemälden gleichzeitig und nebeneinander besteht, ist - soweit ich die Forschung überblicke - zwar vielfach beobachtet, aber bisher nicht befriedigend erklärt worden. Friedrichs in den Quellen fassbare Kritik an einem durch das Bild vorgegebenen Illusionismus, also an dem klassisch-perspektivischen, sprich dreidimensionalen Bildraum, wie er seit der Renaissance in der Kunstgeschichte entwickelt worden war, ist jedoch evident.

Es ist wahrscheinlich, dass Friedrichs Protestantismus, genauer sein Luthertum, ihn sowohl einerseits eine Skepsis gegenüber dem illusionsgebundenen Bildraum wie auch andererseits eine fast manische Bildtreue gegenüber dem mimetischen Detail der Naturdinge entwickeln ließ. Dass daraus eine Perspektive entstand, die das Auge des Künstlers und des Betrachters zur entscheidenden Instanz machte, das hat in der lutherischen Auffassung seine Ursache, dass keinerlei Instanz, keine Kirche, keine weltliche Macht zwischen dem einzelnen Gläubigen und dem Gotteswerk stehen dürfe, die interpretatorisch vermitteln und beeinflussen könnte. Andererseits sind aber auch epistemologische Gründe mit der subjektiven Perspektive verbunden, wie sie Chladenius beschreibt und wie sie sich im 18. Jahrhundert allmählich durchsetzt. Es gibt also verschiedene „SehePunkt[e] von ein und derselben Sache“ und, daraus resultierend, Personen, die

55 Johann Martin Chladenius: Allgemeine Geschichtswissenschaft. Leipzig 1752, Neudruck Wien, Köln, Graz 1985, S. 100 ff.

56 Vgl. ebd. 
eine Sache aus verschiedenen Sehe-Punkten ansehen und somit auch verschiedene Vorstellungen von ihr haben.

Von daher gibt es bei den Friedrichschen Bildern - zumindest aus theologischer und auch aus wissenschaftsgeschichtlicher Perspektive - wohl keine Alternative zu einer mehrdeutig angelegten Interpretation. Die Schwestern auf dem Söller sind bei aller Dislokation der dargestellten Orte eben deshalb ,lesbar', weil (nur) ein lutherischer Bildbetrachter in der Lage ist, diese Diskrepanz der vielen, in einem Bild versammelten Orte wahrzunehmen und in seiner eigenen subjektiven Betrachtung in ein schlüssiges Ganzes zu verwandeln. Die Bindung der Illusion an das „sentiment“ wird bei Friedrich umgedeutet, indem er die Illusion nicht mehr als trügerische Vorgabe des Bildes konstruiert, also wie beim traditionellen perspektivischen Bildraum mit einem einzigen Fluchtpunkt, sondern an dem Scheincharakter des Bildes generell keinen Zweifel mehr lässt. Der perspektivische Fluchtpunkt wird so aus dem Bild in den gläubigen Rezipienten hineingelegt, in den durchaus distanziert vor dem Bild stehenden Künstler oder den Betrachtenden, der die Perspektive auf sich selbst zu beziehen und damit zu vollenden hat. 